\title{
Post-operative imaging surveillance of breast cancer patients status post lumpectomy with BioZorb implant placement
}

\author{
Shruthi Ram ${ }^{1 *}$, Eric Dietsche ${ }^{1}$ \\ 1. Department of Diagnostic Imaging, Rhode Island Hospital, Providence, USA \\ * Correspondence: Shruthi Ram, MD, Rhode Island Hospital/ The Warren Alpert Medical School of Brown University, 593 Eddy St, \\ Providence, RI 02903, USA \\ (入s shruthi.ram@lifespan.org)
}

Radiology Case. 2021 Feb; 15(2):7-24 :: DOI: 10.3941/jrcr.v15i2.3911

\begin{abstract}
The evolving nature of newer surgical techniques and devices adds to the challenge of interpreting breast imaging in the post lumpectomy setting. The BioZorb surgical marker placed at lumpectomy sites for post-operative radiation targeting has its own distinct imaging appearance on each modality. Determining the expected follow-up imaging findings unique to this relatively new device is an ongoing endeavor with minimal literature on this topic. The following series of cases of post BioZorb breast surveillance are one of the first reported in the literature to provide examples of developing asymmetries and new or enlarging masses adjacent to the BioZorb site along with the final pathologic diagnosis for each case. Pathology findings range from fat necrosis and foreign body reaction to recurrent disease.
\end{abstract}

\section{CASE SERIES}

\section{CASE SERIES}

\section{Case 1}

A 49-year-old female had a screening mammogram detected $5 \mathrm{~mm}$ mass in the left breast upper outer quadrant, subsequently biopsy proven to be invasive ductal carcinoma, grade 1, ER+PR+HER2neu-. The patient then underwent left breast lumpectomy with BioZorb implant placement, a negative left sentinel lymph node biopsy, followed by postoperative radiation. Imaging surveillance of the post lumpectomy breast was performed on a 6-month basis. On the left breast surveillance mammogram performed at 2.5 years post lumpectomy, a new mass along the inferior aspect of the BioZorb site in the axillary tail was observed with sonographic correlate (Figure 4). Biopsy of the mass revealed "foreign materials, histiocyte aggregates, giant cells and lymphocytes."
This was deemed concordant with imaging findings. A 6 month follow up mammogram ordered by the patient's surgeon revealed post-operative and post biopsy findings and no mammographic evidence of malignancy.

\section{Case 2}

A 64-year-old female had a screening mammogram detected $2.6 \mathrm{~cm}$ mass in the right breast upper outer quadrant, subsequently biopsy proven to be invasive mucinous carcinoma, grade 1-2, ER+PR+HER2neu-. Patient then underwent right breast lumpectomy with BioZorb implant placement, a negative sentinel node biopsy, followed by postoperative radiation. Imaging surveillance at 2 years post lumpectomy shows a new density at the posterior aspect of the absorbed BioZorb device (Figure 5). On additional views, it was felt to be of low density, probably fat necrosis. Ultrasound 
showed an echogenic mass correlating with mammographic findings. Stereotactic biopsy was performed and showed amorphous material, cyst wall and a focus of fat necrosis, which was deemed concordant with imaging findings.

\section{Case 3}

A 55-year-old female had a screening mammogram finding of a $1.1 \mathrm{~cm}$ mass at 12 o'clock position in the left breast, subsequently biopsy proven invasive ductal carcinoma, grade 2, ER+PR+HER2neu-. Patient then underwent lumpectomy with BioZorb implant placement, a re-excision due to positive superior margin, negative sentinel lymph node biopsy and postoperative radiation. Imaging surveillance at 3 years post lumpectomy showed a new low-density mass forming anterior to the BioZorb site (Figure 6). Ultrasound showed several evolving areas of fat necrosis. Stereotactic biopsy showed fat necrosis, which was deemed concordant with imaging.

\section{Case 4}

A 67-year-old female had a screening mammogram finding of a $2.7 \mathrm{~cm}$ mass at 12 o'clock position in the left breast, biopsy proven to be invasive ductal carcinoma, grade 2, ER+PR+HER2neu-. Patient then underwent a left breast lumpectomy with BioZorb implant placement. Sentinel lymph nodes were negative for malignancy. Patient also underwent postoperative radiation. Imaging surveillance of the postoperative breast was performed on a 6-month basis. On the left breast surveillance mammogram performed 3.5 years after surgery, a new asymmetry in the left breast at the 12 o'clock position was noted at the inferior aspect of the resorbing BioZorb (Figure 7). Stereotactic biopsy was recommended, which was performed and showed fat necrosis with adjacent fibrosis and scar tissue. This was deemed concordant with imaging findings. A 6-month post biopsy follow up was recommended.

\section{Case 5}

A 53-year-old female had a screening mammogram finding of a $9 \mathrm{~mm}$ spiculated mass at 11 o'clock position in the left breast, biopsy proven to be invasive ductal carcinoma, grade 2, ER+PR+HER2neu-. Patient then underwent a lumpectomy with BioZorb implant placement, a negative sentinel lymph node biopsy followed by postoperative radiation. Imaging surveillance of the post-operative breast was performed on a 6-month basis. On the left breast surveillance mammogram performed 2.5 years after surgery, a new $1.8 \mathrm{~cm}$ circumscribed mass was identified lateral and superior to the BioZorb implant at 12 o'clock (Figure 8). Ultrasound guided biopsy was recommended, which was performed and showed fat necrosis. This was deemed concordant with imaging findings and a 6 month follow up mammogram was recommended.

\section{Case 6}

A 55-year-old female had a screening mammogram finding of a $1.1 \mathrm{~cm}$ mass in the 11 o'clock area of the left breast, biopsy proven to be ductal carcinoma in situ (DCIS), micropapillary and cribriform types, grade II, ER+. She subsequently underwent a lumpectomy with BioZorb placement and postoperative radiation therapy. Surveillance mammography was performed on a 6-month basis. Mammogram 3 years after surgery showed development of small masses adjacent to the BioZorb (Figure 9). Stereotactic biopsy was performed, showing fat necrosis, which was deemed concordant with imaging findings.

\section{Case 7}

A 58-year-old woman presented with a pathology report from the Dominican Republic demonstrating invasive mammary carcinoma. Imaging performed at our institution demonstrated an $8 \mathrm{~mm}$ mass at 7 o'clock in the right breast, subsequently biopsied and proven to be invasive ductal carcinoma, grade 3, ER-PR-HER2neu-. The patient then underwent a right breast lumpectomy with BioZorb placement and negative sentinel lymph node biopsy. Patient also underwent chemoradiation therapy. Imaging surveillance of the post-surgical breast was performed on a 6-month basis, with a mammogram 3 years post op showing a new focal asymmetry in the right breast adjacent to the BioZorb (Figure 10). Biopsy showed fibrosis, with associated foreign-body material and sheets of histiocytes. This was deemed concordant with imaging findings.

\section{Case 8}

A 75-year-old female had a prior history of left breast DCIS status post breast conservation therapy. Her left breast was then followed on a 6-month basis and her right breast annually. Her right-sided annual mammogram at 3 years out from her initial cancer diagnosis showed a $6 \mathrm{~mm}$ irregular mass at the 1:00 position, right breast. Biopsy showed invasive ductal carcinoma, grade II, ER+PR+HER2neu-equivocal. Patient then underwent right breast lumpectomy with BioZorb implant placement, a negative sentinel lymph node biopsy and postoperative radiation. Imaging surveillance was then performed on a 6-month basis. On the right breast surveillance mammogram performed at 3 years post lumpectomy, a new 3 mm mass was seen at 2:00 position medial to BioZorb site with sonographic correlate. Biopsy of the $3 \mathrm{~mm}$ mass showed invasive ductal carcinoma, grade II, ER+PR+HER2neu-. A focal asymmetry was also noted posterior to the BioZorb site, which persisted on additional views. However, ultrasound was limited in evaluation of this deep location posterior to BioZorb site due to shadowing from the device and scar tissue (Figure 11). An MRI was recommended for the focal asymmetry posterior to BioZorb site, which showed a $2.0 \mathrm{~cm}$ suspicious mass in this region (Figure 11). Patient underwent stereotactic biopsy of the mammographic correlate, which showed additional site of disease. Patient then underwent right mastectomy.

\section{DISCUSSION}

The imaging findings of the post-lumpectomy breast may present as a diagnostic challenge for many reasons. The distortion and fat necrosis related findings at the surgical scar site can occasionally confound image interpretation. The evolving nature of newer surgical techniques and devices can add to the challenge, making it especially important to stay well-informed on associated imaging findings. For example, 
the BioZorb surgical marker placed at lumpectomy sites for post-operative radiation targeting has its own distinct imaging appearance on each modality[1]. Determining the expected follow-up imaging findings unique to this relatively new device is an ongoing endeavor with limited literature on this topic.

The BioZorb marker is an oncoplastic device implanted within the tumor resection bed. The original device measures about $2-3 \mathrm{~cm}$ and consists of 6 radiopaque titanium clips secured by a bioabsorbable helix (Figure 1). The bioabsorbable portion of the device is expected to slowly absorb within the body over 1-2 years. The purpose of the device is to provide a more standardized method of marking the lumpectomy bed. The idea is to allow for more precise targeting in postoperative radiation treatment[2] and enhanced visual confirmation in post-operative imaging surveillance.

Mammography is the modality of choice in the surveillance of the post-lumpectomy breast in patients with a history of breast cancer[3,4]. At our institutions, the BioZorb device was used for a brief period of time. The post BioZorb breast has its own unique expected imaging findings on mammogram, ultrasound and MRI (Figure 2). Over time, as the device absorbs, convergence of the radiopaque titanium clips is observed mammographically (Figure 3).

Some of the key imaging findings of recurrence in the post lumpectomy breast include a developing asymmetry or enlarging mass at the post-operative site, regardless of whether BioZorb is used. Our series of cases of post BioZorb breast surveillance are one of the first reported in the literature that provide examples of developing asymmetries and new or enlarging masses adjacent to the BioZorb site along with the final pathologic diagnosis for each case.

\section{Etiology \& Demographics:}

BioZorb associated densities can be seen in female breast cancer patients status post breast conservation therapy with BioZorb device placement at the surgical site.

\section{Clinical \& Imaging findings:}

Literature on imaging of the post lumpectomy breast after BioZorb implant placement is sparse. A previous case series showcasing mammographic follow up appearance of the post BioZorb breast concluded that mammographic imaging follow-up was not adversely affected by the device[5,6]. Our institutional experience has seen a substantial volume of surveillance imaging in the post BioZorb setting. In our experience, the post BioZorb breast has presented challenges in post-operative surveillance image interpretation, some of which may be specific to the device. As the device absorbs and convergence of titanium markers is observed, vague nonspecific densities from adjacent tissue ingrowth and fibrosis may develop around the post-operative site.

In addition, we have observed the time to absorption of the device to be quite variable. Time to absorption of the device has ranged from the initially expected $1-2$ years post placement with absorption sometimes delayed to 4-5 years out from placement. Occasional calcification of the residual bioabsorbable helix portion of the device has also been observed (Figure 12). We have also evaluated the postBioZorb breast in a variety of clinical scenarios including symptoms of pain, palpable mass and skin changes (Figures 13, 14). The use of BioZorb at our institution was subsequently stopped due to concerns regarding these patient symptoms post BioZorb placement. Even in the absence of symptoms, the occasional presence of BioZorb associated mammographic densities on surveillance follow-up imaging has created diagnostic challenges, necessitating biopsies in some cases.

The differential diagnosis for a BioZorb associated density primarily includes fat necrosis or breast cancer recurrence. Some key differentiating features between these two entities on mammogram involve careful assessment of the density and margins of the mass. In fat necrosis, developing masses on mammogram tend to be of low density with circumscribed margins and occasionally with characteristic rim calcification in cases of oil cyst formation. Recurrent disease on mammogram typically presents as a high density mass with indistinct margins. However, unless the mass is clearly fat-containing and radiolucent with classic rim calcification, it can be hard to make the diagnosis of fat necrosis on imaging alone and subsequent pathologic confirmation is usually necessary.

Making the diagnosis of fat necrosis on ultrasound can be more challenging than on mammogram, due to its typical heterogenous appearance with areas of mixed echogenicity superimposed on shadowing scar tissue. Our cases demonstrate the limitation of ultrasound in the post BioZorb breast due to pronounced shadowing caused by the device, which may lead to diagnostic challenges. In cases of equivocal findings or technically difficult biopsy, MRI may be helpful as a problem-solving tool in the evaluation of the post-surgical site (Figure 15), especially beyond 18 months after surgery[7,8]. The diagnosis of fat necrosis can sometimes be made definitively on MRI when a fat intensity mass is seen with minimal rim enhancement. On the other hand, recurrence on MRI presents as an irregular mass or nodular enhancement at the lumpectomy site.

\section{Treatment \& Prognosis:}

In a patient population with a prior recent history of treated breast cancer, need for biopsy of the surgical site can lead to significant patient anxiety and stress. In most cases, due to limitations of ultrasound in evaluating the post BioZorb breast, stereotactic biopsy is usually required, which is more difficult and uncomfortable for patients. Findings from this case series support the need for tissue sampling in patients with new or developing BioZorb densities as in any case of increased density of a lumpectomy site However, when being counseled for the biopsy procedure, patients may be offered reassurance that in many cases, BioZorb related densities can occur from benign causes, with recurrence being a relatively rare cause. In addition, in cases of adequate target sampling, a benign result of fat necrosis or foreign body reaction should be considered concordant and the patient may then return to routine follow-up. 


\section{Differential Diagnoses:}

Benign causes: Fat necrosis/foreign body reaction/fibrosis

The most common cause of a BioZorb associated developing density in the breast is related to fat necrosis or foreign body fibrotic reaction. It appears that the breast tissue adjacent to the BioZorb reacts to the device, resulting in soft tissue densities or masses forming at the lumpectomy site.

\section{Malignant causes: Breast cancer recurrence}

Although less common, breast cancer recurrence remains in the differential in the setting of a developing focal asymmetry adjacent to the BioZorb site.

\section{Conclusion}

As demonstrated in our case series, the finding of increased density or mass associated with BioZorb site may represent benign entities such as foreign body reaction and fat necrosis or malignancy. In most cases of the indeterminate imaging finding of developing density, tissue sampling is necessary and recommended for definitive diagnosis to exclude the infrequent but existing possibility of locoregional recurrent disease.

\section{TEACHING POINT}

Key imaging findings of recurrence in the post lumpectomy breast include a developing asymmetry or enlarging mass at the post-operative site, which has a differential diagnosis of fat necrosis. Interpretation of the post lumpectomy breast can be challenging, especially with the evolving nature of newer surgical techniques and devices.

\section{REFERENCES}

1. Ward RC, Wiggins DL, Donegan L, Collins S, Lourenco AP, Mainiero MB. BioZorb tissue marker as seen on multiple imaging modalities. Breast J 2018;24(2):207-209. PMID: 28805318

2. Foster B, Sindhu K, Hepel J, et al. Three-Dimensional Bioabsorbable Tissue Marker Placement is Associated with Decreased Tumor Bed Volume Among Patients Receiving Radiation Therapy for Breast Cancer. Pract Radiat Oncol 2019;9(2):e134-e141. PMID: 30268431

3. Lam DL, Houssami N, Lee JM. Imaging Surveillance After Primary Breast Cancer Treatment. AJR Am J Roentgenol 2017;208(3):676-686. PMID: 28075622

4. Lewin AA, Moy L, Baron P, et al. ACR Appropriateness Criteria ${ }^{\circledR}$ Stage I Breast Cancer: Initial Workup and Surveillance for Local Recurrence and Distant Metastases in Asymptomatic Women. J Am Coll Radiol 2019;16(11S):S428-S439. PMID: 31685110

5. Harms S, Lebovic G, Kaufman C, Cross M. Mammographic imaging after partial breast reconstruction: Impact of a bioabsorbable breast implant. J Clinic Oncol 33 (suppl 28S; abstr 111). PMID: 28147836

6. Cross MJ, Lebovic GS, Ross J, Jones S, Smith A, Harms S. Impact of a Novel Bioabsorbable Implant on Radiation Treatment Planning for Breast Cancer. World J Surg 2017;41(2):464-471. PMID: 27709273

7. Drukteinis JS, Gombos EC, Raza S, Chikarmane SA, Swami A, Birdwell RL. MR imaging assessment of the breast after breast conservation therapy: distinguishing benign from malignant lesions. Radiographics 2012;32(1):219-34. PMID: 22236903

8. Chansakul T, Lai KC, Slanetz PJ. The postconservation breast: part 2, Imaging findings of tumor recurrence and other long-term sequelae. AJR Am J Roentgenol 2012;198(2):33143. PMID: 22268175

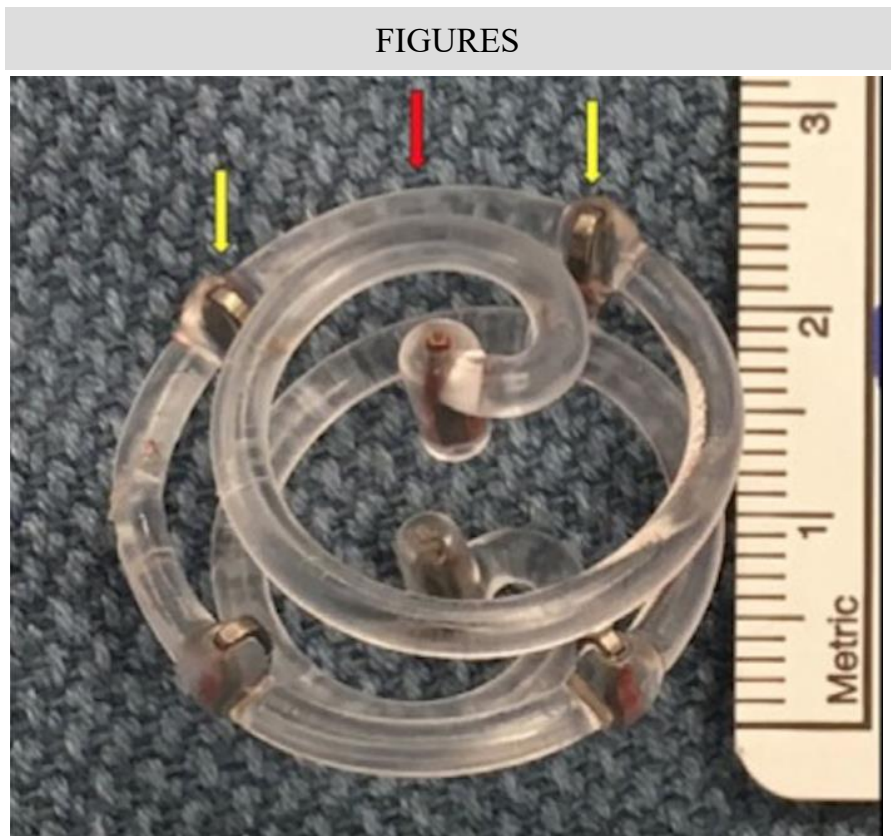

Figure 1: $2.5 \mathrm{~cm}$ BioZorbTM (Focal Therapeutics, Aliso Viejo, CA, USA) tissue marker. Note the six titanium clips (two of six marked by two yellow arrows) bound by the bioabsorbable spiral (red arrow). 


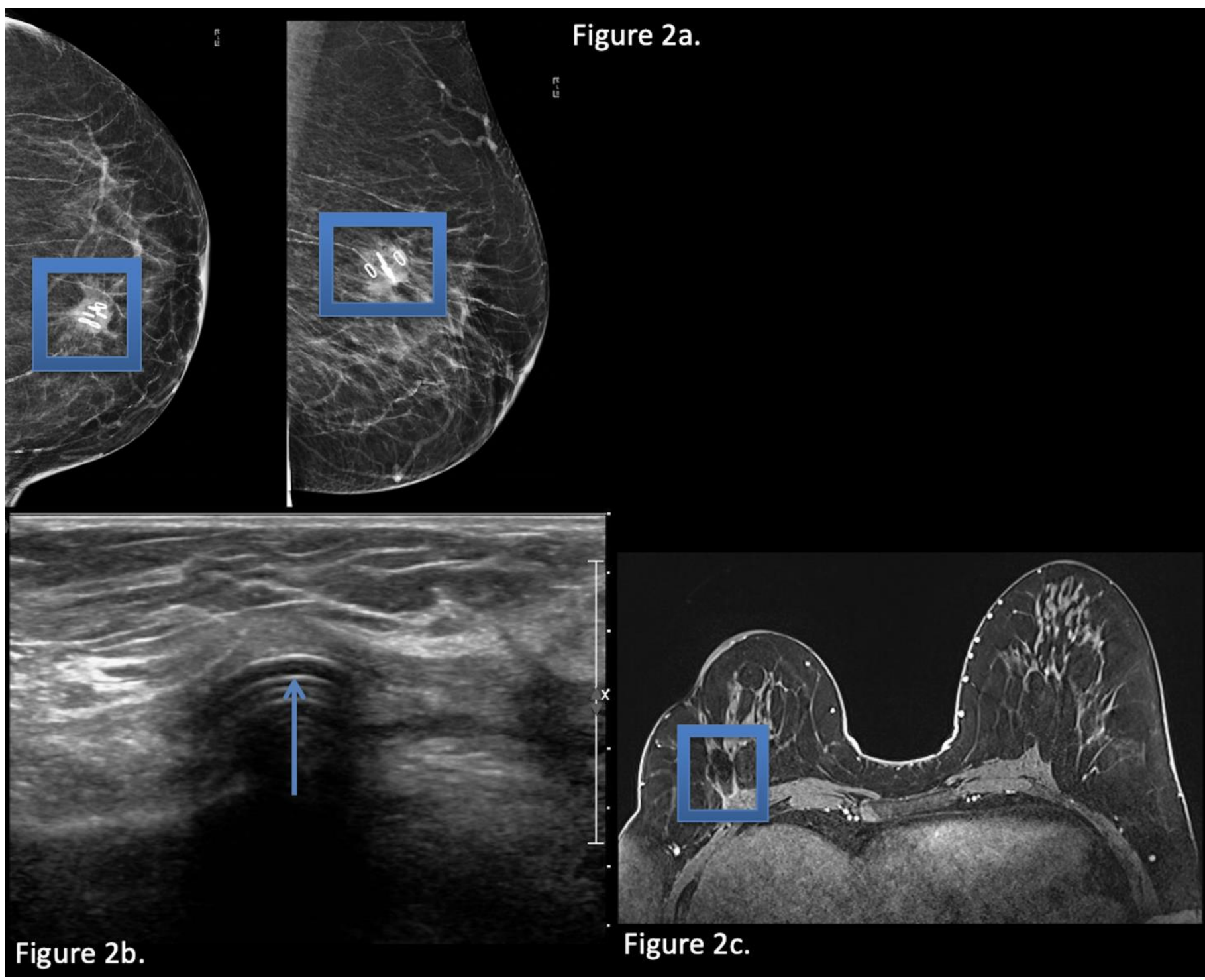

Figure 2: 53-year-old female with normal post-BioZorb breast. She had a history of left breast cancer status post breast conservation therapy with BioZorb placement and underwent postoperative surveillance imaging:

(a) Technique: 2D screening mammogram (kVp: 33, mAs: 210), Craniocaudal (CC) and Mediolateral oblique (MLO) views of the right breast.

Findings: Normal appearance of BioZorb (annotated), showing radioopaque titanium markers.

56-year-old female with normal post-BioZorb breast. She had a history of right breast cancer status post breast conservation therapy with BioZorb and underwent postoperative surveillance imaging:

(b)

Technique: Targeted ultrasound of the breast, using a $12 \mathrm{MHz}$ Linear array transducer (Phillips) in transverse plane.

Findings: Normal appearance of BioZorb. Note the tubular bioabsorbable ring of the device (arrow) and the pronounced posterior acoustic shadowing.

(c)

Technique: Breast MRI axial post contrast T1-weighted sequence with fat saturation. Performed on a $1.5 \mathrm{~T}$ magnet, $16.4 \mathrm{~mL}$ of gadoterate meglumine administered, TR 3.76, TE 1.5.

Findings: Normal appearance of BioZorb site, with associated susceptibility artifact and minimal peripheral enhancement (annotated). No suspicious finding such as mass or nodular enhancement is present. 


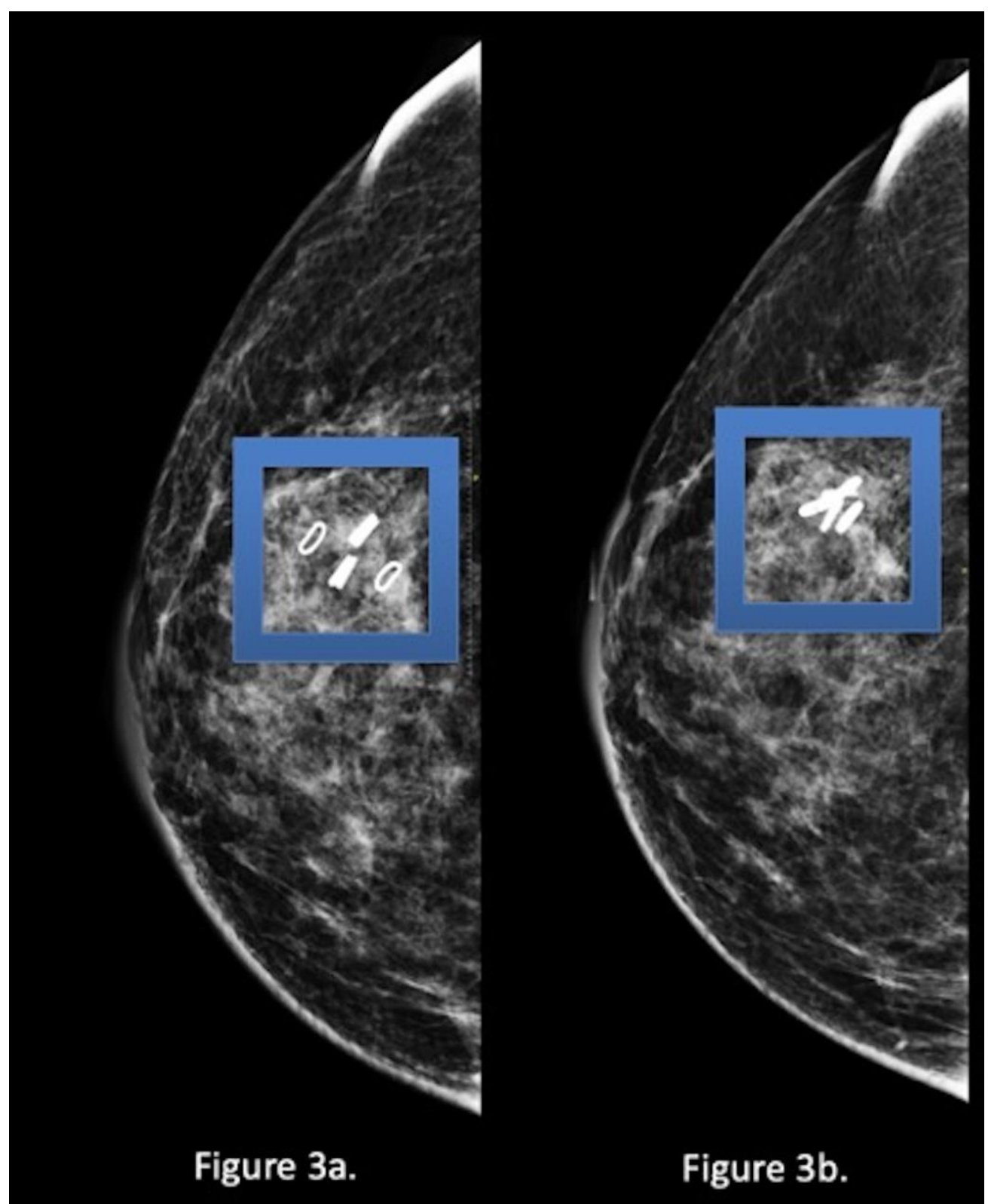

Figure 3: 65-year-old female with normal post-BioZorb breast. She had a history of right breast cancer status post breast conservation therapy with BioZorb.

Technique: 2D screening mammogram; CC views of prior (a) (kVp 31, mAs: 264) and current study (b) (kVp:32, mAs: 266).

Findings: Convergence of BioZorb radiopaque markers due to absorption of device. (a) Baseline post-operative study 6 months after surgery. (b) Imaging 3 years after surgery shows absorption of device, as demonstrated by convergence of the titanium markers. No abnormal post-surgical site density is seen. 


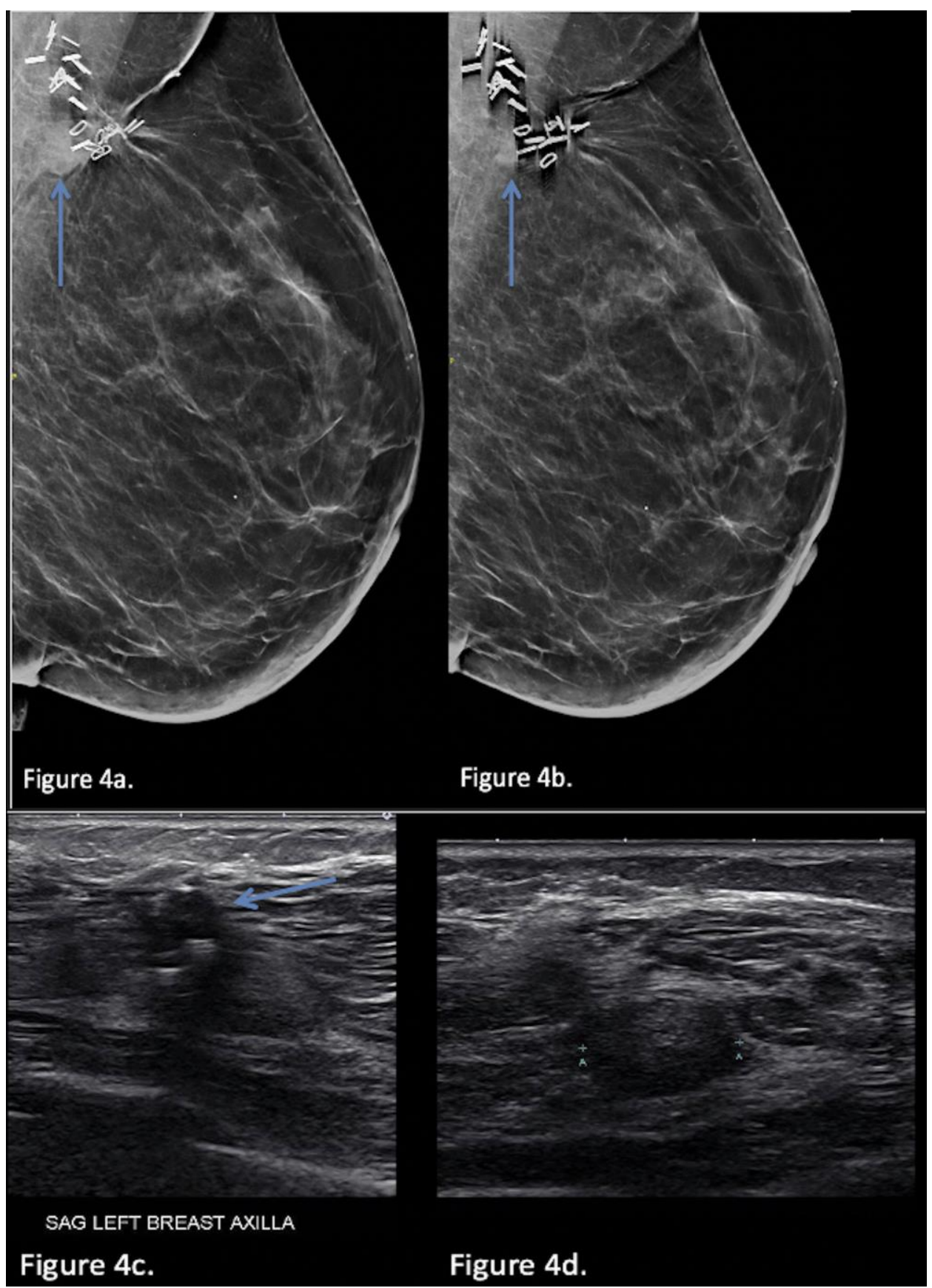

Figure 4: 49-year-old female with a BioZorb associated developing asymmetry, with subsequent biopsy results yielding foreign materials and giant cells.

(a-b)

Technique: Diagnostic mammogram with Synthetic 2D MLO views of current (a) (kVp: 39, mAs: 95) and prior (b) (kVp: 38, mAs: 97) study.

Finding: Asymmetry at the inferior aspect of BioZorb lumpectomy site (arrow in a), increased since previous study from one year prior (arrow in b).

(c-d)

Technique: Targeted ultrasound of the BioZorb site in the left breast using an $18 \mathrm{MHz}$ linear array transducer (Toshiba Aplio 500), in sagittal plane.

Findings: BioZorb (arrow in c) and an oval circumscribed hypoechoic mass inferior to it (calipers in d), correlating with the mammographic asymmetry. 


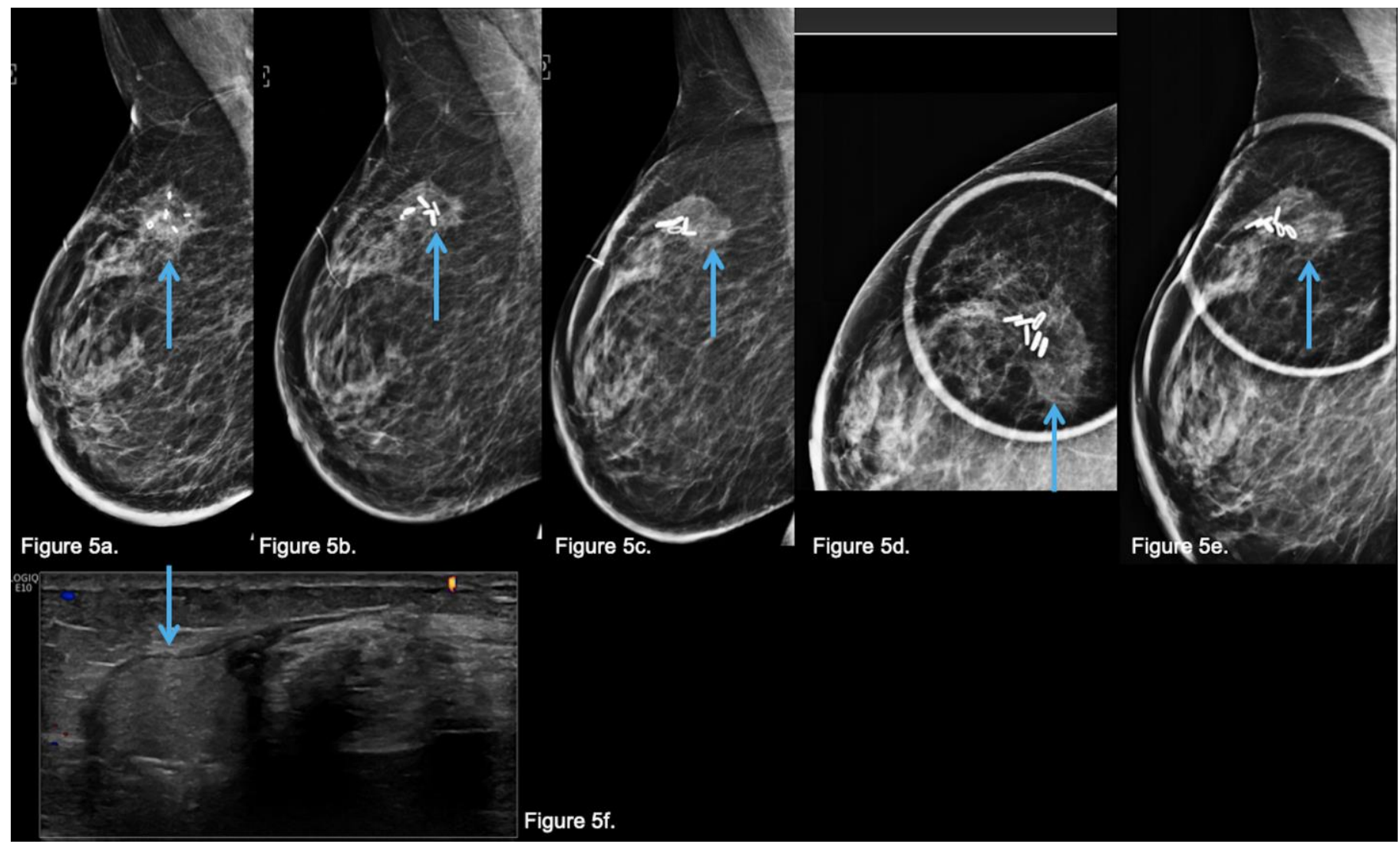

Figure 5: 64-year-old female with a BioZorb associated asymmetry, with subsequent biopsy results yielding fat necrosis.

(a-e)

Technique: Diagnostic 2D mammogram MLO views from baseline post operative (post op) surveillance mammogram (a) (kVp: 28, mAs: 152), 18 months post op (b) kVp: 29, mAs: 150)and 2 years post op (c) (kVp: 28, mAs: 151). Spot compression views in CC (d) and MLO views (e) were also obtained to evaluate the asymmetry further at 2 years post op.

Findings: Gradual absorption of the BioZorb device (arrows in a and b), along with a new low-density spiculated asymmetry (arrows in c through e) at the posterior aspect of the device. (f)

Technique: Targeted ultrasound of the surgical site in the right breast, using a 15MHz linear array transducer (GE Logiq E10), in sagittal plane.

Findings: Echogenic mass (arrow in f) corresponding to the mammographic asymmetry (arrows in c through e). 


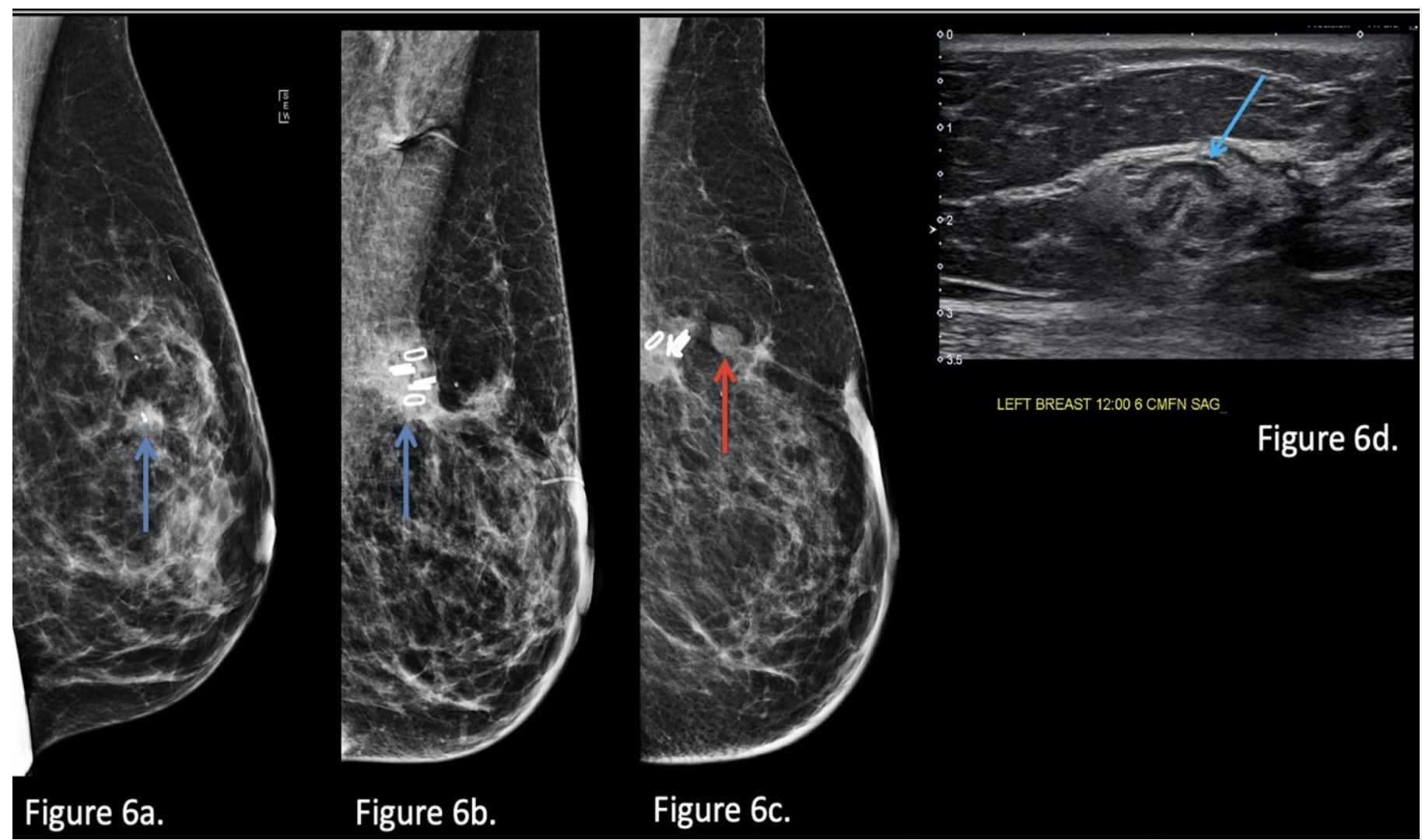

Figure 6: 55-year-old female with biopsy proven left sided invasive ductal carcinoma (a), status post lumpectomy and placement of a BioZorb device (b), with development of a BioZorb associated mass (c) and subsequent stereotactic breast biopsy demonstrating fat necrosis.

$(\mathrm{a}-\mathrm{c})$

Technique: Diagnostic 2D mammogram MLO view obtained preoperatively (a) (kVp: 33, mAs: 205), 1-year post op, (b) (kVp: 32, mAs: 204) and 3 years post op (c) (kVp: 32, mAs: 205).

Findings: Biopsy proven malignant mass (blue arrow in a) with clip in place. Post lumpectomy surveillance mammograms show the BioZorb device (arrow in b) and a new low-density circumscribed mass (red arrow in c) forming anterior to it.

(d)

Technique: Targeted ultrasound of the surgical site in the left breast, using a $14 \mathrm{MHz}$ linear array transducer (Toshiba, Aplio 500), in sagittal plane.

Findings: Area of mixed echogenicity without definitive correlate for the low density mass seen on mammography. Findings were deemed to be suggestive of fat necrosis, and this was proven on subsequent pathology results from stereotactic biopsy. 


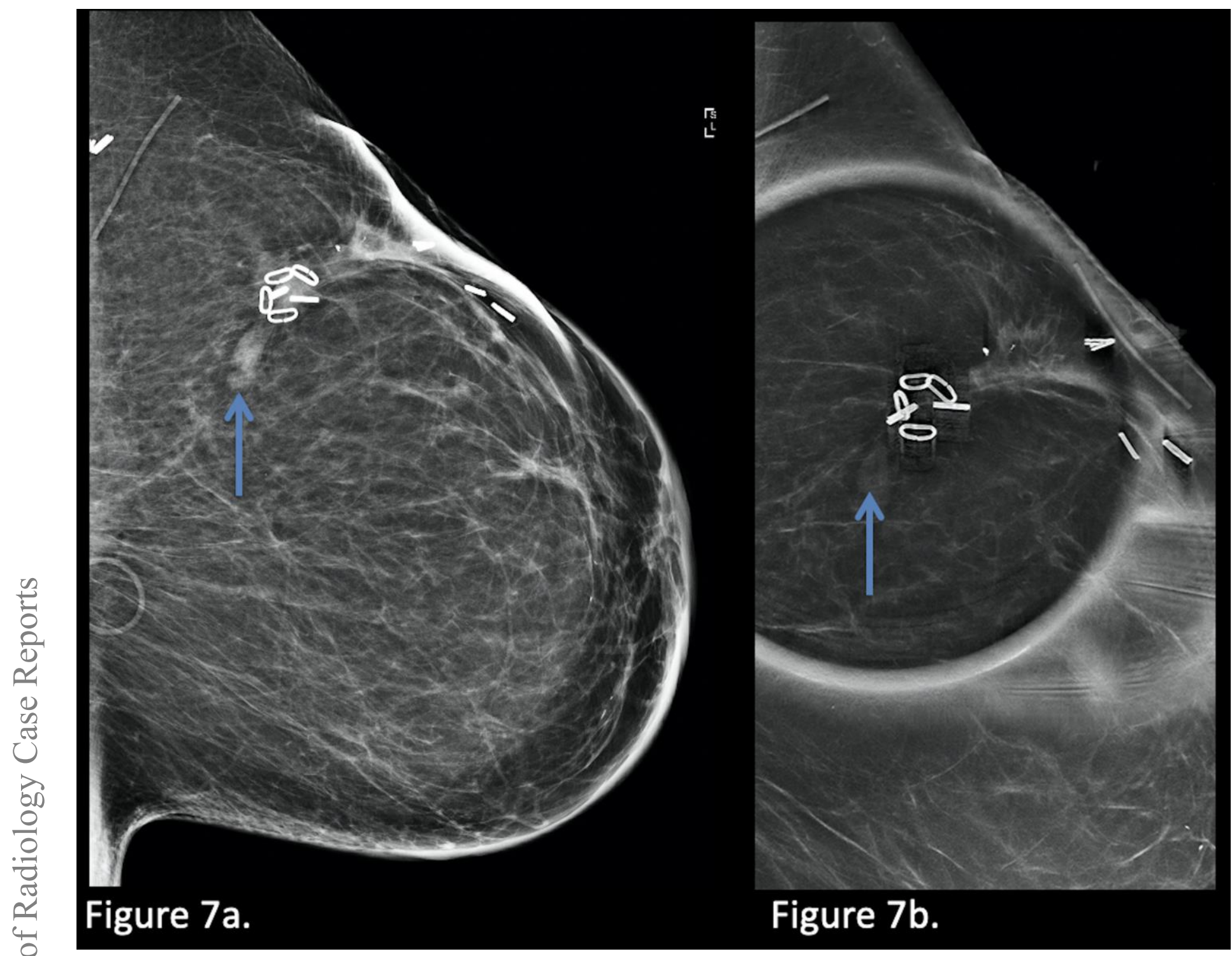

Figure 7: 67-year-old female with a BioZorb associated asymmetry, with subsequent biopsy results showing fat necrosis and fibrosis.

Technique: Diagnostic 2D mammogram Mediolateral (ML) view (a) (kVp: 31, mAs: 115) and Synthetic 2D spot ML view (b) (kVp: 32, mAs: 112)

Findings: Left breast mass with oval shape and circumscribed margins (arrows) inferior to BioZorb site. 


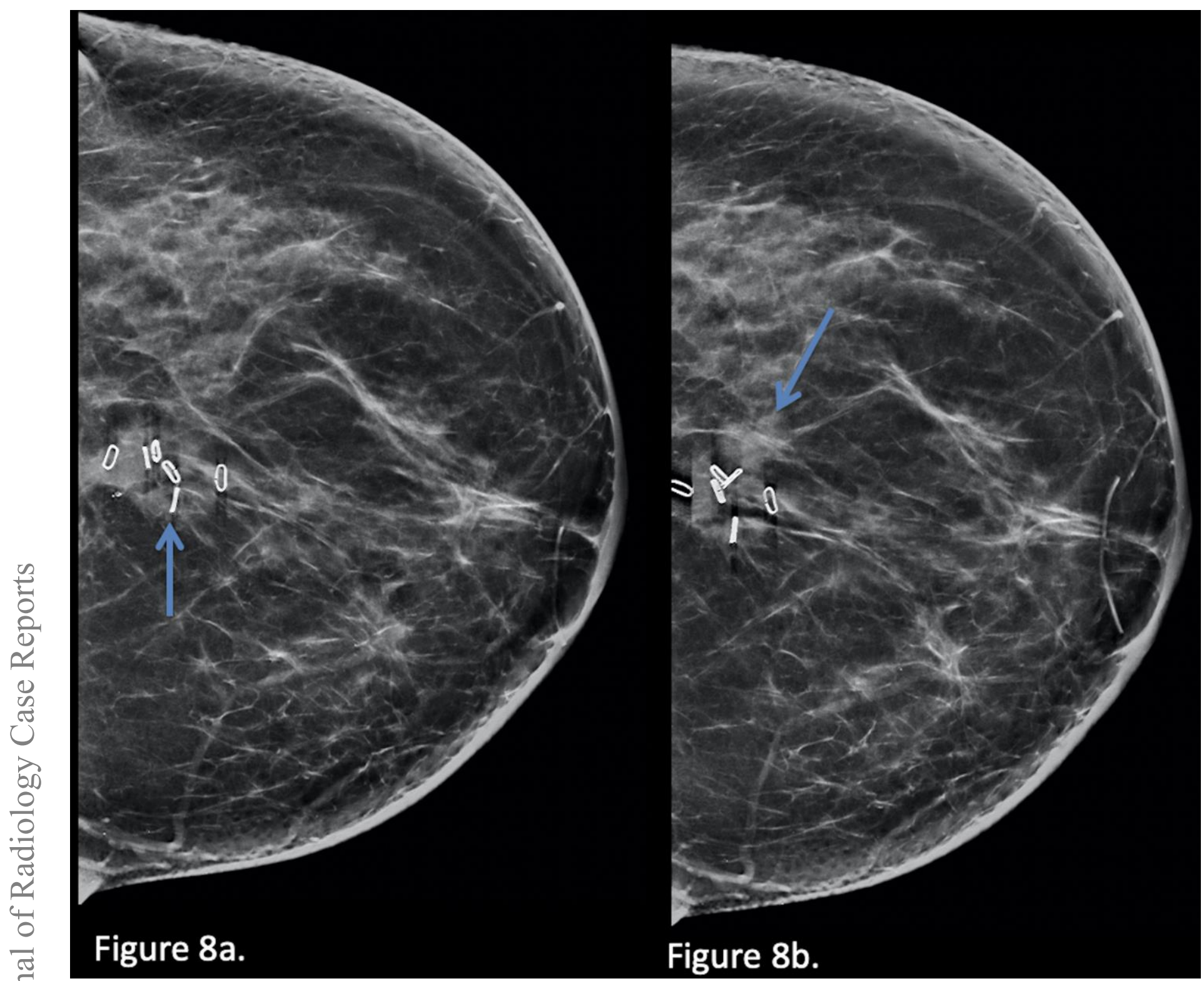

Figure 8: 53-year-old female with a BioZorb associated mass in the left breast, biopsy results of which showed fat necrosis.

Technique: Diagnostic Synthetic 2D mammogram CC view 1-year (a) (kVp: 32, mAs: 90) and 2.5 years (b) post op (kVp: 32, mAs: 92).

Findings: Left breast mass with circumscribed margins (arrow in b) lateral to BioZorb site (arrow in a), new since prior study (a). 


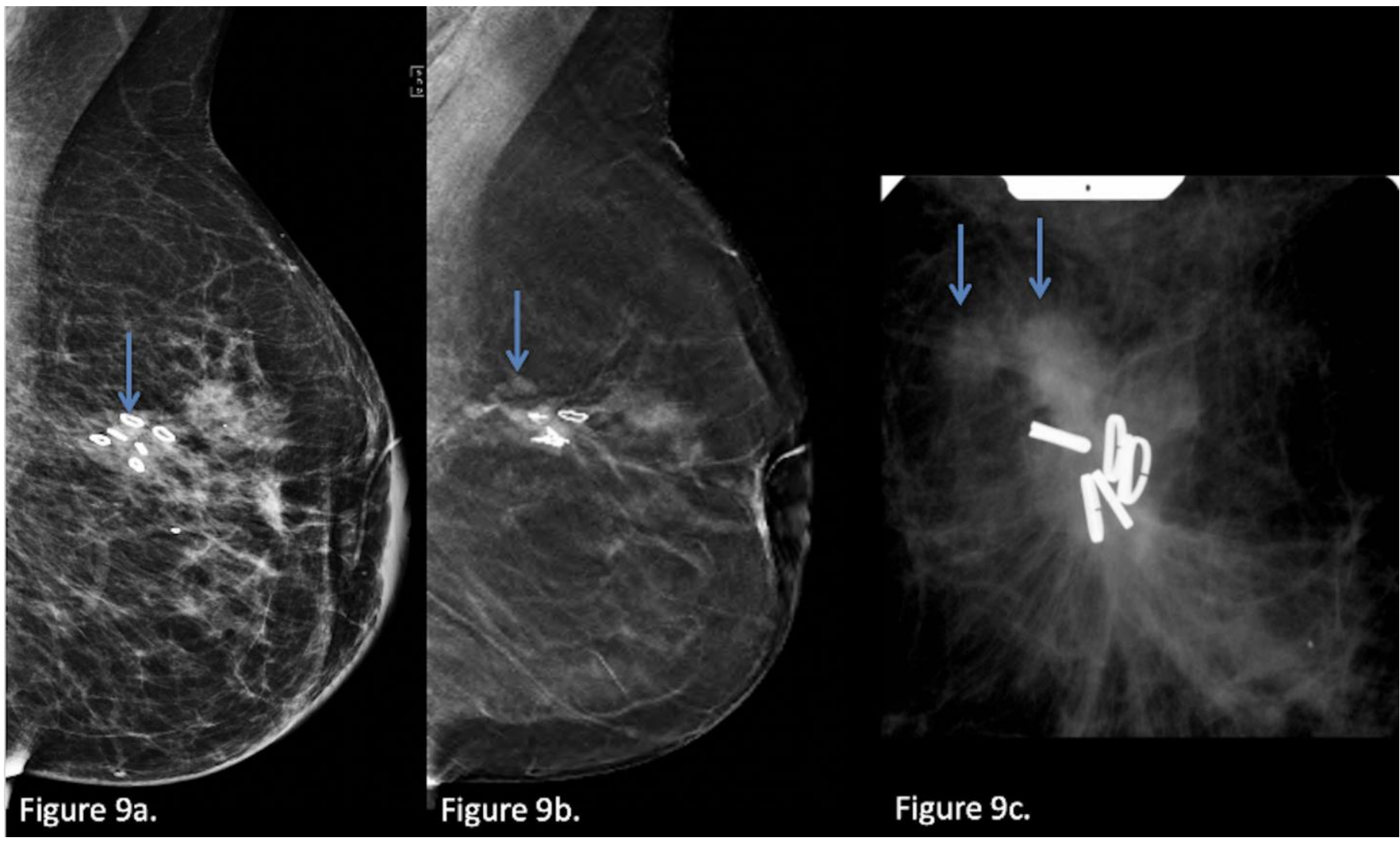

Figure 9: 55-year old female with BioZorb associated masses in the left breast., with biopsy results from stereotactic biopsy showing fat necrosis.

Technique: Diagnostic 2D mammogram MLO view (a) (kVp: 34, mAs: 95) obtained 6 months post op 3D mammogram MLO view single tomosynthesis slice (b) ( $\mathrm{kVp}: 34$, mAs: 93) obtained 3 years post op and 2D mammogram MLO scout view from the stereotactic biopsy (c)

Findings: Development of two circumscribed masses (arrows in b and c) adjacent to BioZorb site (arrow in a), new since prior study (a), subsequently targeted using stereotactic guidance (c). 


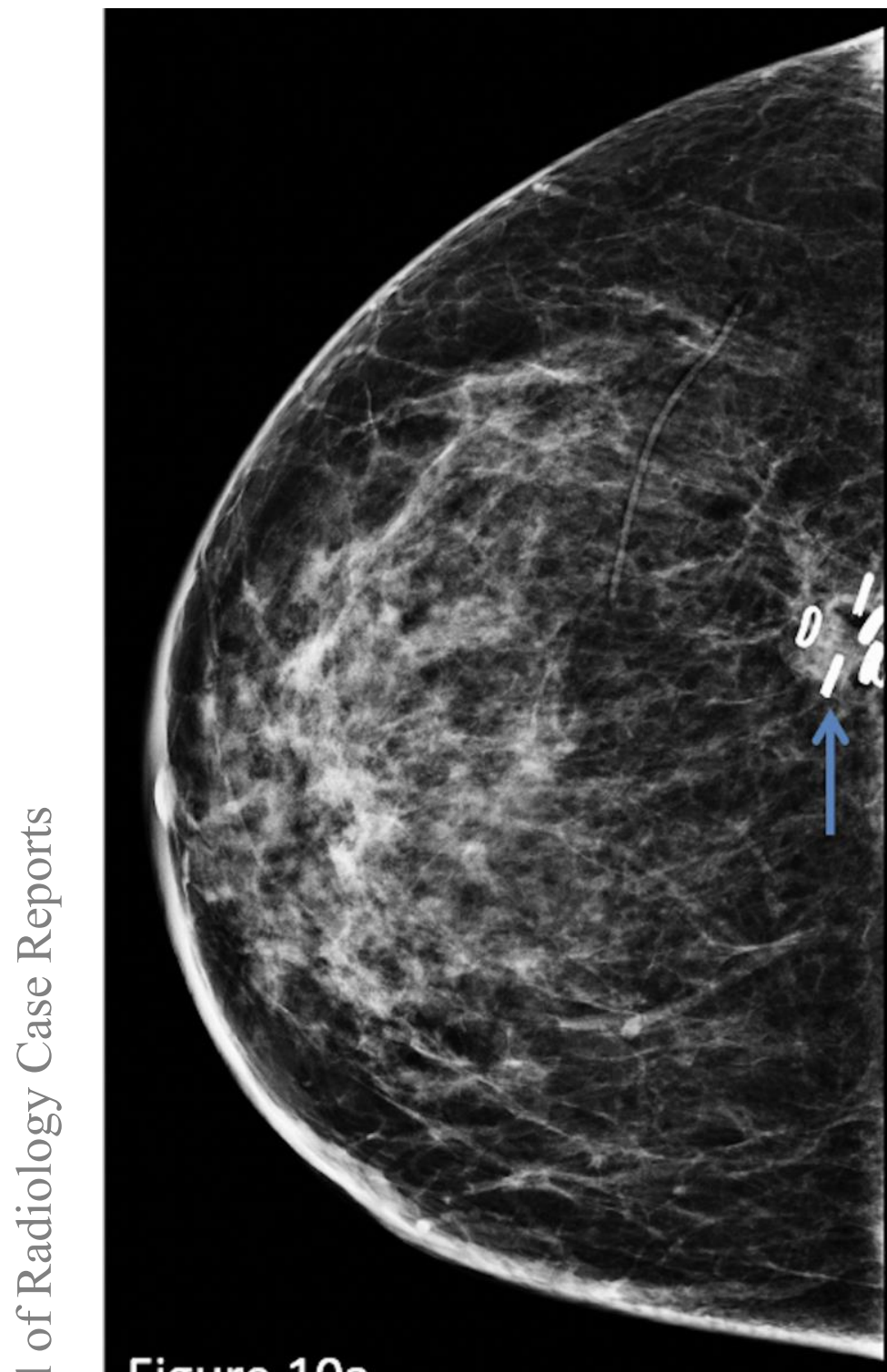

Figure 10a.

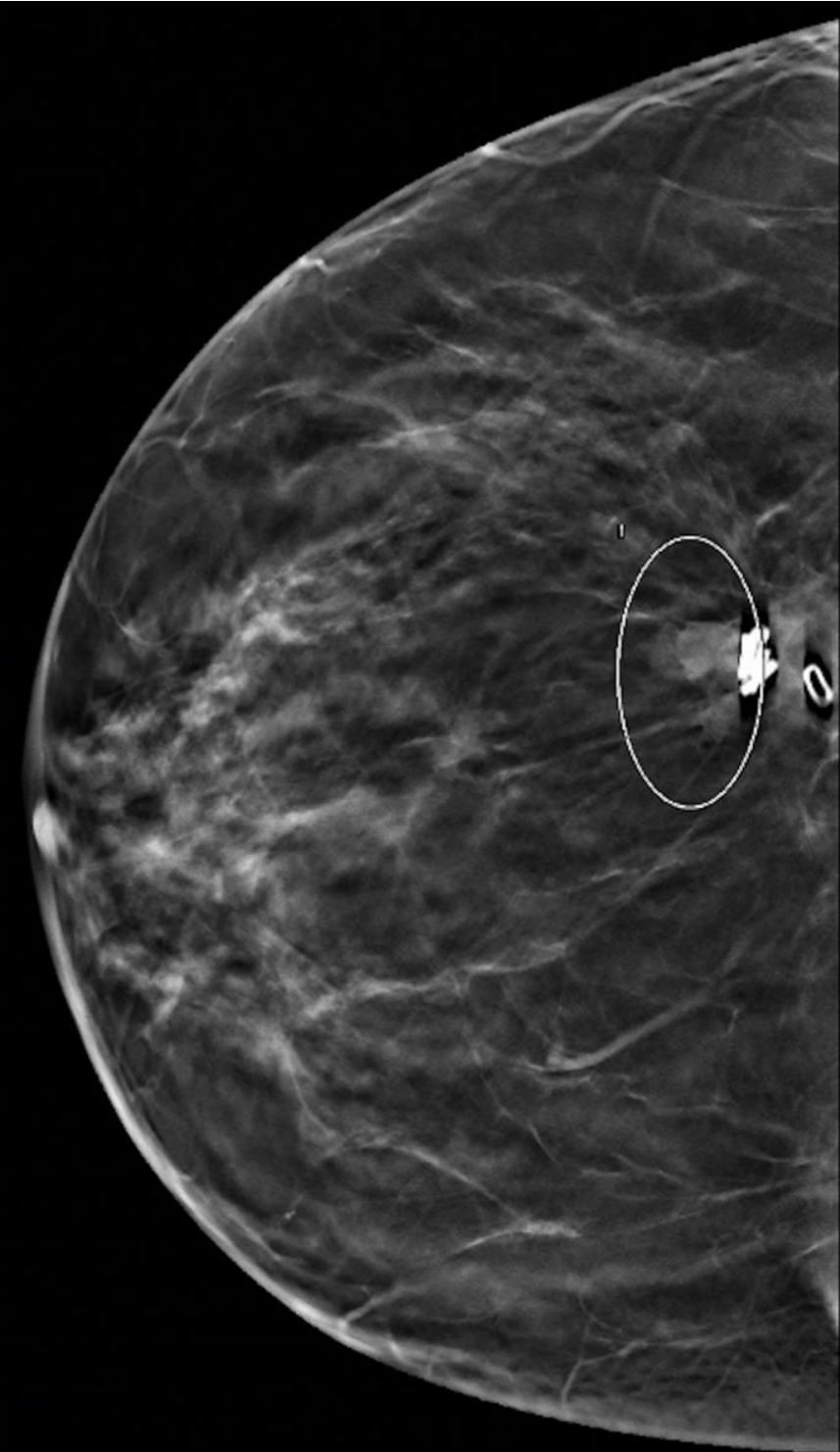

Figure 10b.

Figure 10: 58-year-old female with a BioZorb associated asymmetry and subsequent pathology results showing fibrosis with associated foreign body material.

Technique: Diagnostic 2D mammogram CC view 1-year post op (a) (kVp: 30, mAs: 250) and 3D mammogram CC view single tomosynthesis slice 3 years post op (b) (kVp: 30, mAs: 255)

Findings: BioZorb device (arrow in a) on post op imaging. Circumscribed mass anterior to retracting BioZorb (annotated in b), new since prior study (a). 


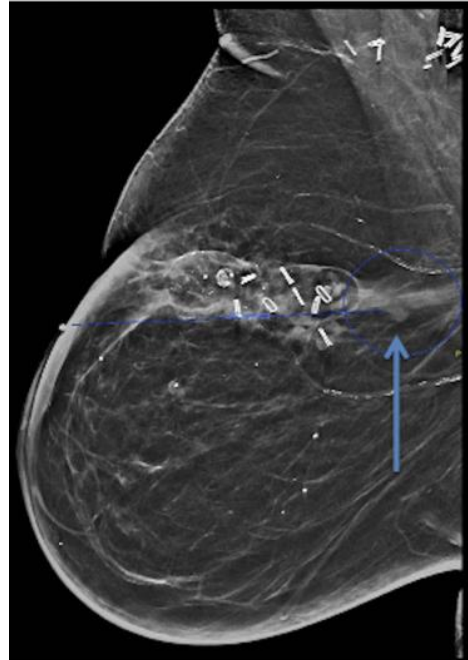

Figure 11a.

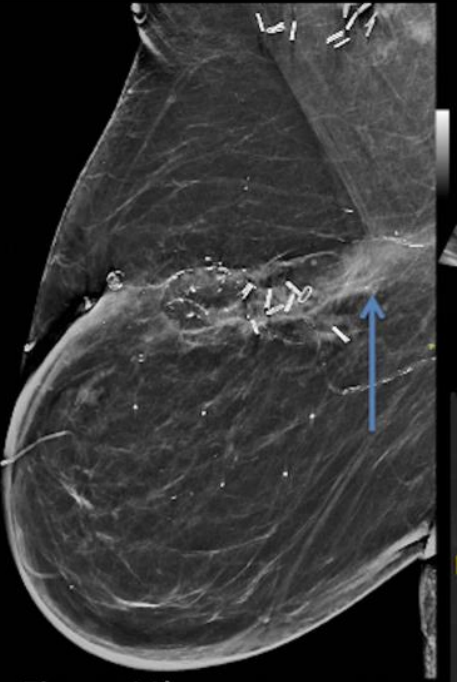

Figure 11b.

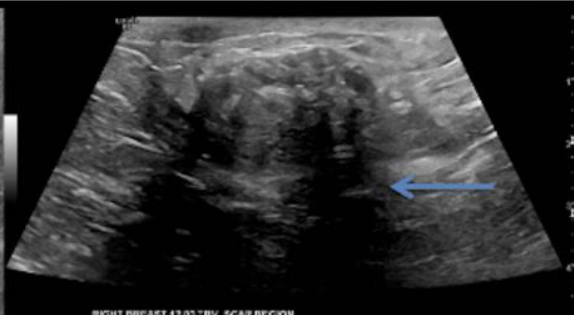

Figure 11c.

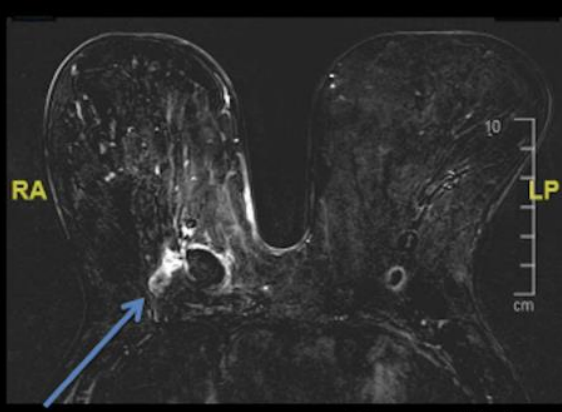

Figure 11d.
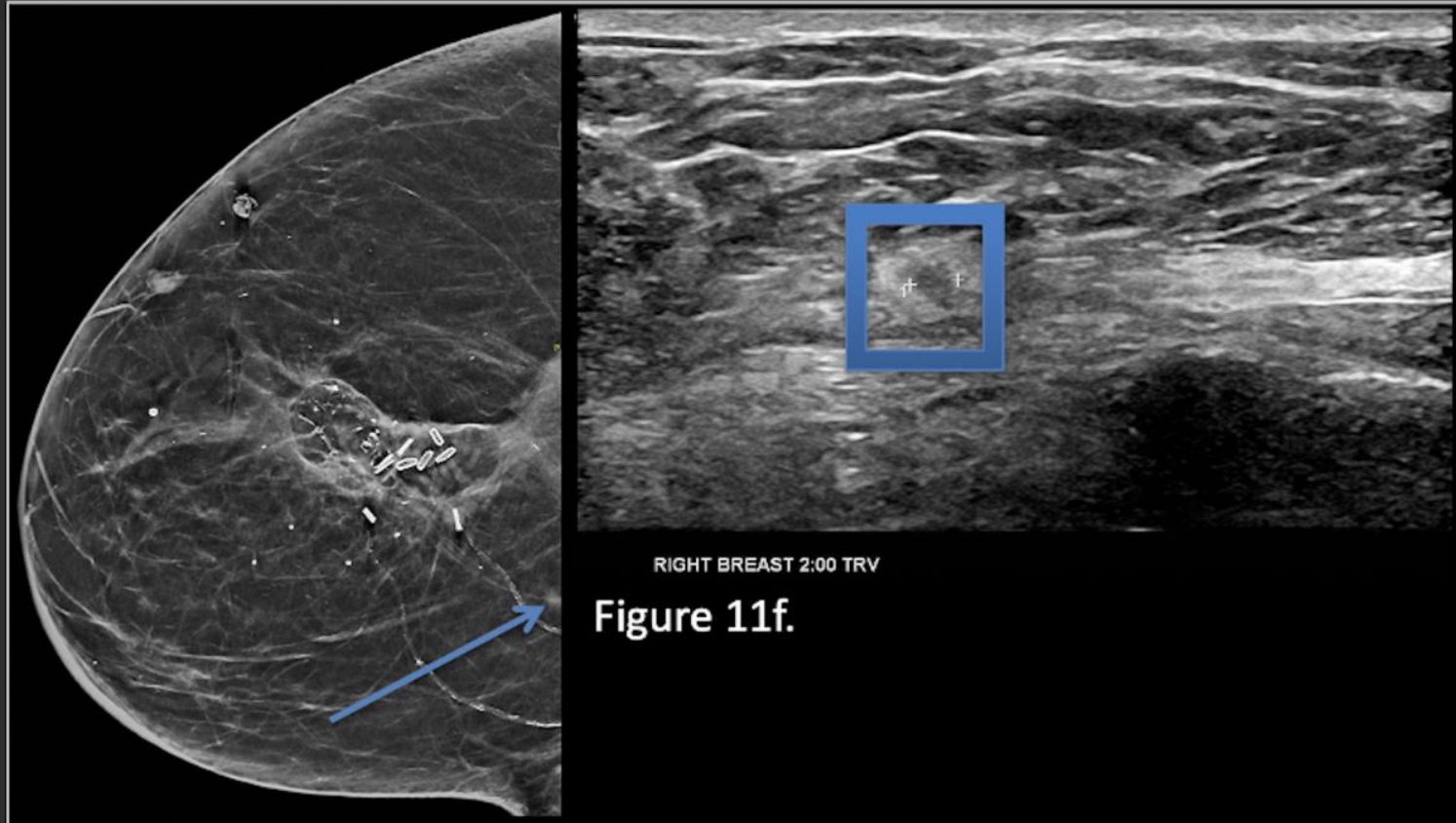

Figure 11f.

\section{Figure 11e.}

Figure 11: 75-year-old female with multifocal breast cancer recurrence after BioZorb placement.

(a-b) Technique: Diagnostic Synthetic 2D mammogram MLO views of the right breast one-year post op (a) (kVp: 34 , mAs: 84 ) and two years post op (b) (kVp: 33, mAs: 84$)$

Findings: Developing focal asymmetry (arrows in a and b) posterior to BioZorb.

(c) Technique: Targeted ultrasound of the surgical site in the right breast, using a $15 \mathrm{MHz}$ linear array transducer (GE Logiq E10), in transverse plane.

Findings: Intense shadowing (arrow in c) from scar and BioZorb limited evaluation for findings posterior to surgical site.

(d) Technique: Breast MRI axial post-contrast T1-weighted subtracted sequence (Performed on a 3T magnet, TR: 4.02, TE: 1.46, Contrast agent: $13 \mathrm{ml}$ gadoterate meglumine)

Findings: There is a $2 \mathrm{~cm}$ irregular enhancing mass (arrow in d) posterior to the BioZorb surgical cavity correlating with the mammographic developing asymmetry. Subsequent stereotactic biopsy results yielded recurrent disease.

(e) Technique: Diagnostic Synthetic 2D mammogram CC view of the right breast two years post op (kVp: 33, mAs: 81)

Findings: $3 \mathrm{~mm}$ irregular asymmetry (arrow in e) medial to and separate from BioZorb site

(f) Technique: Targeted ultrasound of the upper inner quadrant focused on site of mammographic concern, using a $15 \mathrm{MHz}$ linear array transducer, in transverse plane.

Findings: $3 \mathrm{~mm}$ irregular hypoechoic mass (annotated) correlating with mammographic abnormality. Biopsy showed additional site of recurrent disease. 


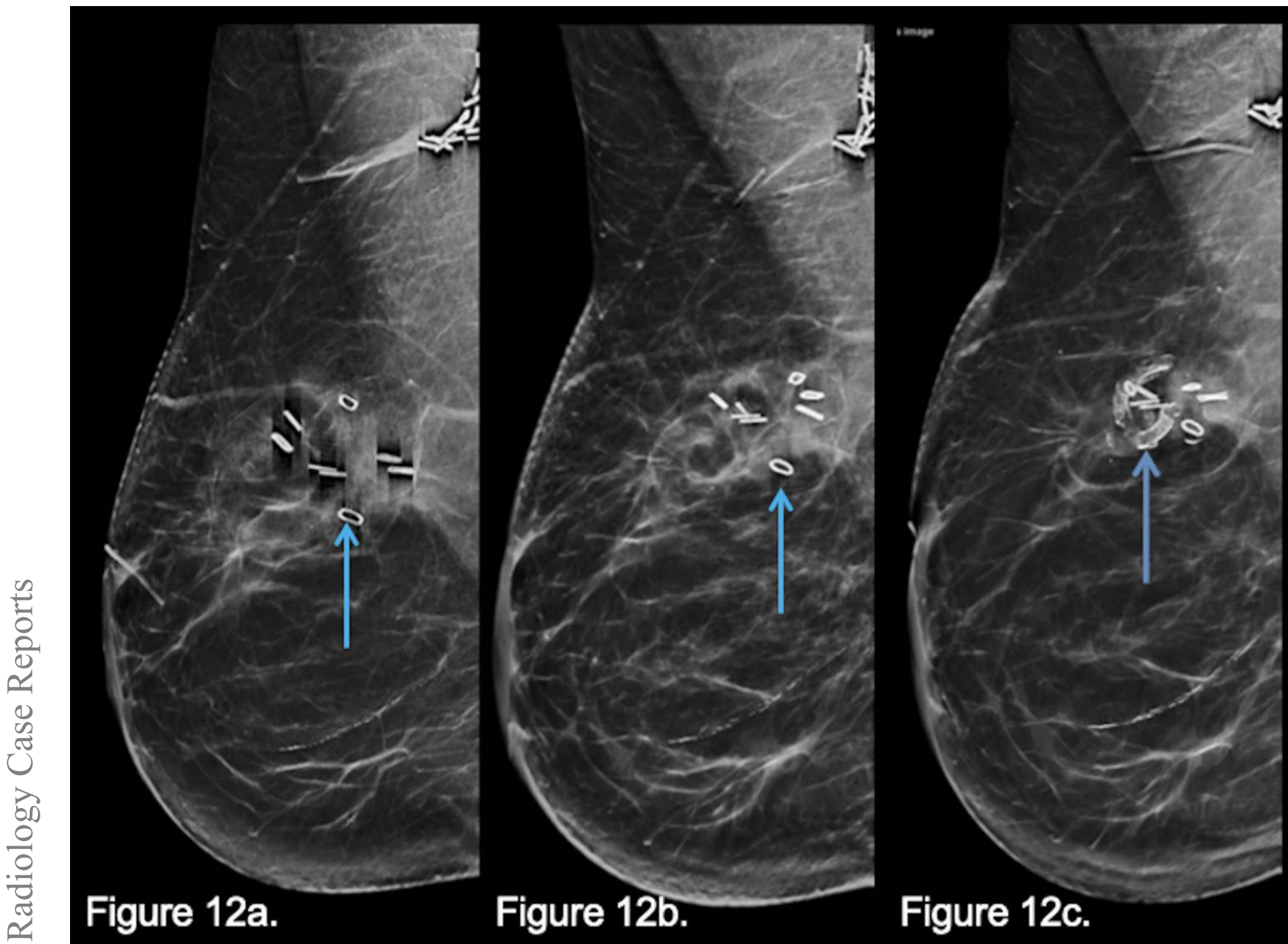

Figure 12: 55-year-old female with BioZorb calcification. She had a history of right breast cancer status post breast conservation therapy with BioZorb who underwent postoperative surveillance imaging:

Technique: Screening Synthetic 2D mammogram MLO views 6 months post op (a) (kVp: 30, mAs: 205), one-year post op (b) (kVp: 30, mAs: 206) and 2 years post op (c) (kVp: 31, mAs: 205)

Findings: Gradual partial absorption of BioZorb - the titanium markers (arrows in a and b) slowly converge, with calcification of residual tubular bioabsorbable portion of the device (arrow in c). 


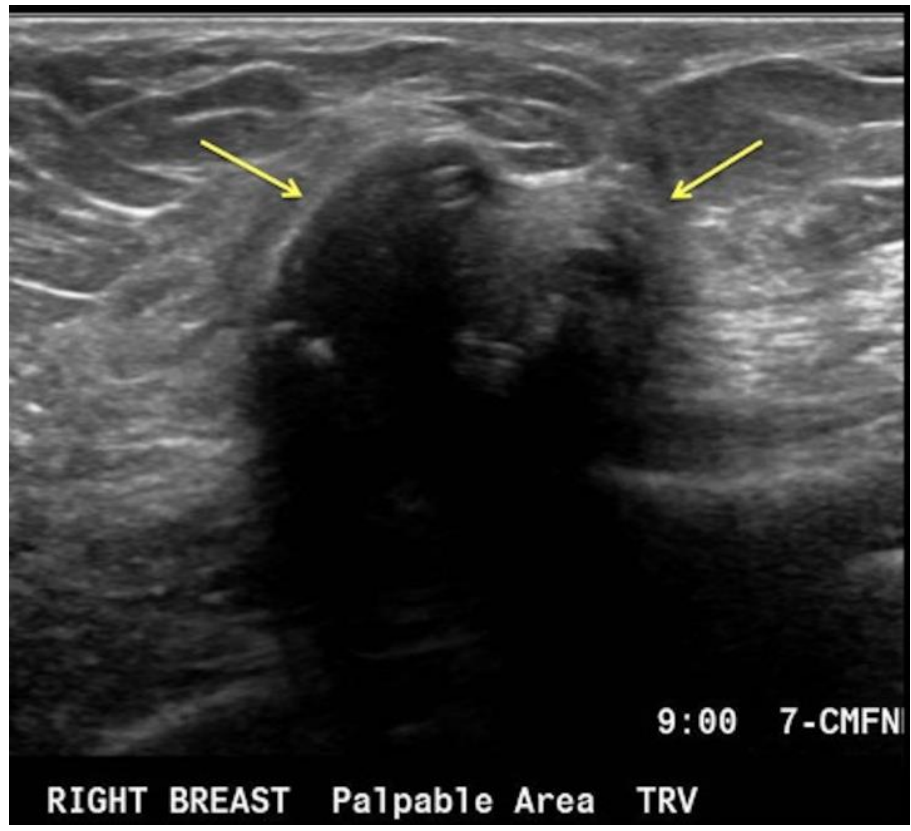

Figure 13 (left): 56-year-old female with palpable lump and normal post-BioZorb breast. She was status post right breast conservation therapy for breast cancer with BioZorb device placement, who presented with a palpable lump at the surgical site 6 months post op.

Technique: Targeted ultrasound of the palpable area of concern in the right breast, using a $12 \mathrm{MHz}$ linear array transducer (Phillips), in transverse plane.

Findings: The BioZorb device itself (arrows) corresponds with the palpable lump. The device appears as a shadowing mass with parts of the bioabsorbable tubular portion visible superiorly.

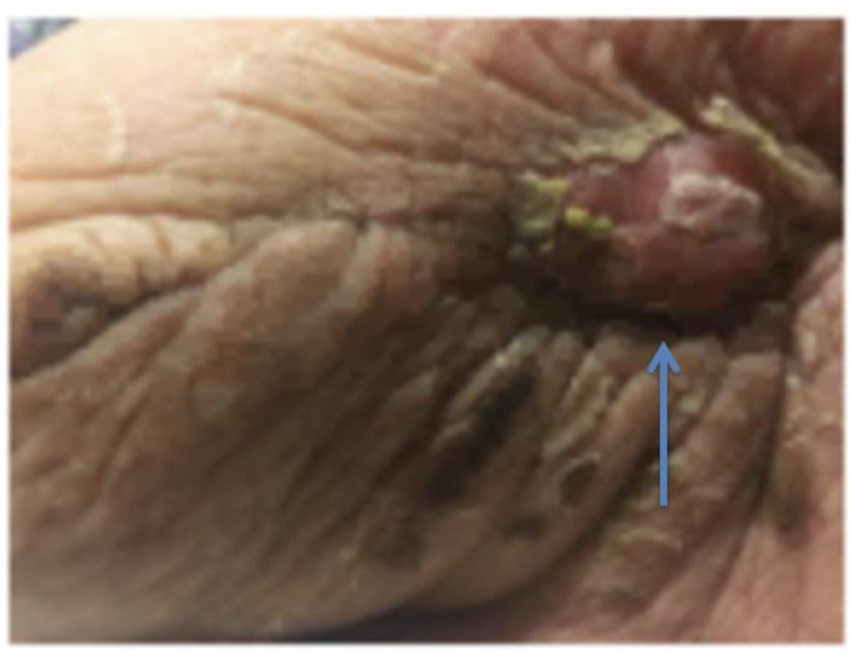

Figure 14a.

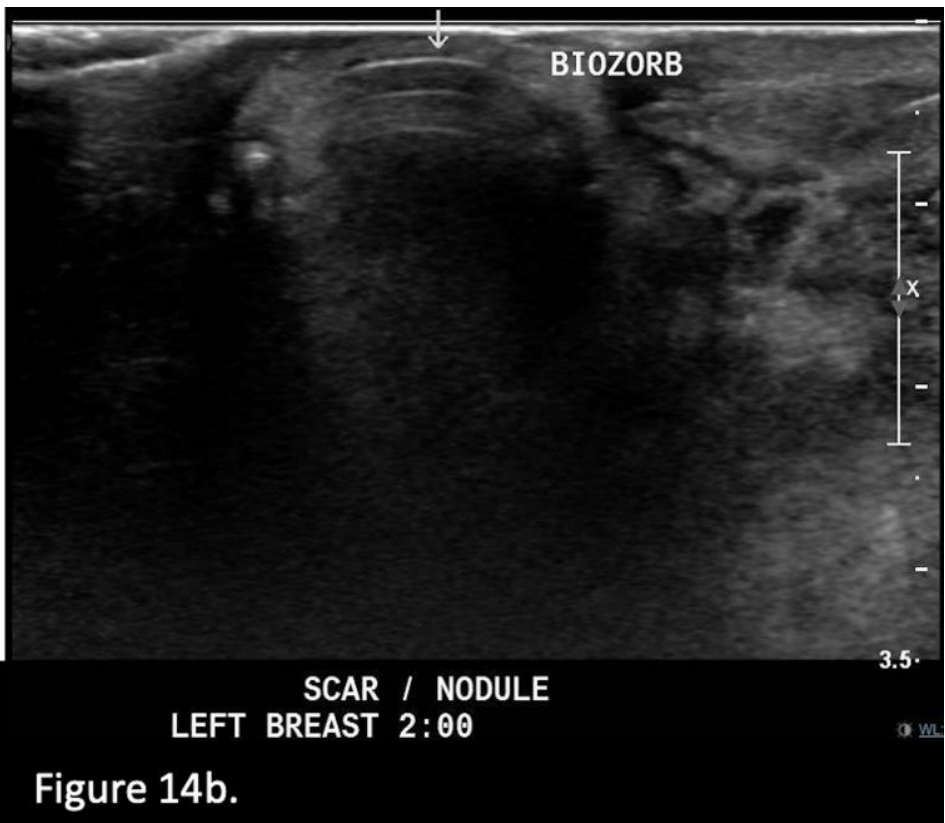

Figure 14b.

Figure 14: 72-year-old female with wound dehiscence and infection status post left breast conservation therapy for breast cancer with BioZorb device placement. She presented with a palpable firm lump with erosive skin changes at the surgical site 6 months post op.

Technique: Photograph (a) and Targeted ultrasound (12 MHz Linear array transducer, Phillips) (transverse plane) of the palpable area of concern in the left breast (b).

Findings: The BioZorb device has eroded through the skin (blue arrow in a) resulting in an open wound. Ultrasound confirms that the BioZorb device has eroded through the skin (white arrow in b). 


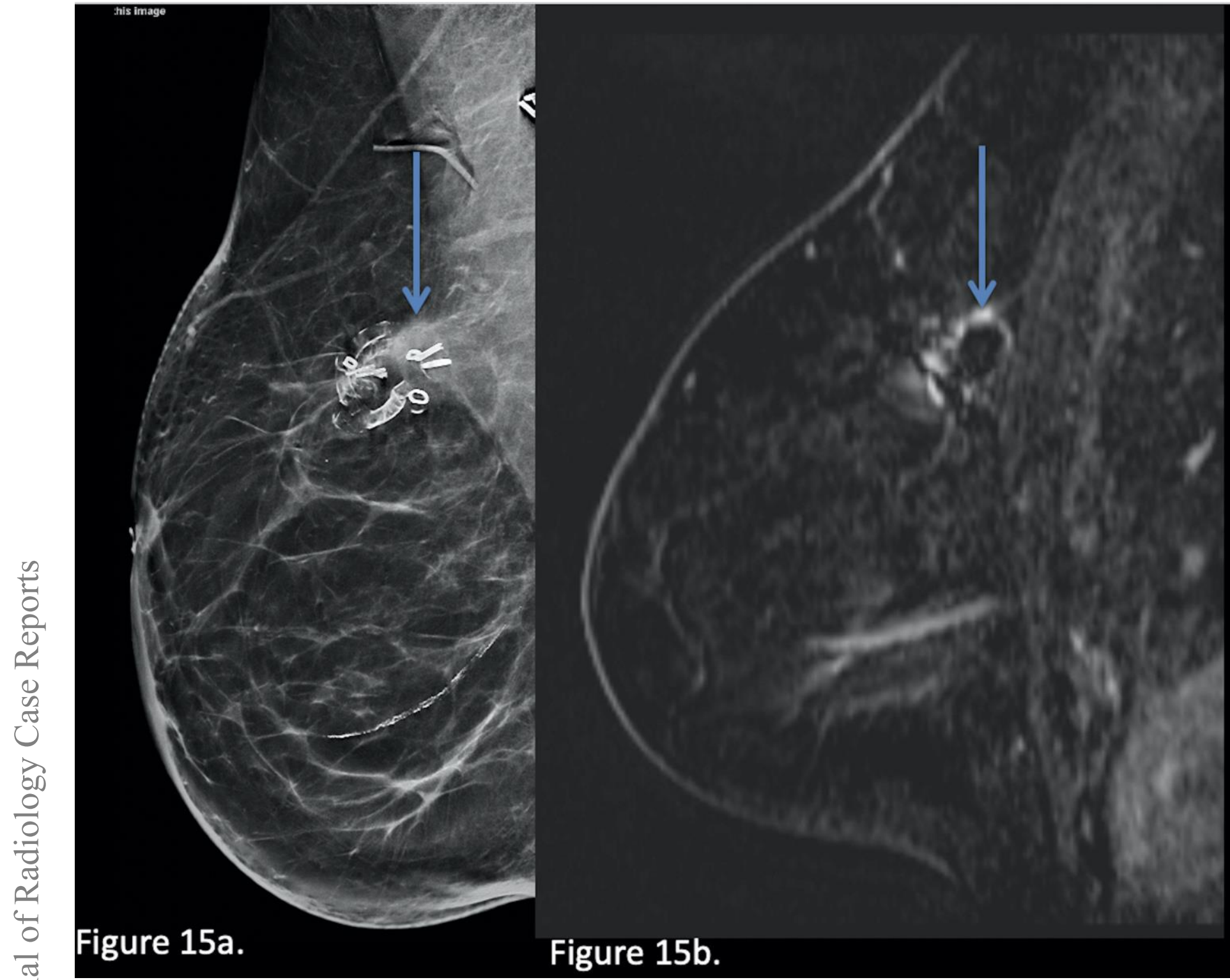

Figure 15: 63-year-old female with a BioZorb associated asymmetry.

Technique: Diagnostic Synthetic 2D mammogram MLO view obtained 3 years post op (a) (kVp: 31, mAs: 205) and subsequent Breast MRI (b) Sagittal post contrast T1-weighted subtracted sequence. Performed on a 3T magnet; $15 \mathrm{~mL}$ of gadoterate meglumine administered. TR 4, TE 1.44.

Findings: Concern of increased density (arrow in a) at the superior aspect of BioZorb site. This was then recommended for stereotactic biopsy, however the density was not seen on the day of the biopsy and MRI was recommended. MRI showed normal mild peripheral enhancement of surgical site (arrow in b) without evidence of suspicious mass or nodular enhancement to suggest recurrence. 


\begin{tabular}{|l|l|}
\hline $\begin{array}{l}\text { Etiologies of BioZorb } \\
\text { associated density }\end{array}$ & Fat necrosis, foreign body reaction, fibrosis, recurrence \\
\hline Incidence & Unknown \\
\hline Gender ratio & N/A \\
\hline Age predilection & Unknown \\
\hline Risk factors & Prior BioZorb device placement \\
\hline Treatment & Biopsy, reassurance, management of recurrence \\
\hline Prognosis & Generally good \\
\hline Imaging findings & Developing density or mass on mammogram with or without sonographic correlate \\
\hline
\end{tabular}

Table 1: Summary table of BioZorb implant associated density on Mammography.

\begin{tabular}{|l|l|l|l|}
\hline & Mammogram & Ultrasound & MRI \\
\hline $\begin{array}{l}\text { Benign causes } \\
\text { (primarily fat necrosis) }\end{array}$ & $\begin{array}{l}\text { Usually low-density mass, } \\
\text { calcifications may be present }\end{array}$ & $\begin{array}{l}\text { Areas of mixed echogenicity at } \\
\text { site of surgical scar }\end{array}$ & $\begin{array}{l}\text { Rim enhancing mass with } \\
\text { central fat intensity }\end{array}$ \\
\hline $\begin{array}{l}\text { Breast cancer } \\
\text { recurrence }\end{array}$ & $\begin{array}{l}\text { Persisting mass or density, } \\
\text { new or increasing over time }\end{array}$ & $\begin{array}{l}\text { May be limited due to } \\
\text { shadowing from scar. }\end{array}$ & $\begin{array}{l}\text { Irregular mass or nodular } \\
\text { enhancement at surgical site }\end{array}$ \\
\hline
\end{tabular}

Table 2: Differential diagnosis table for lumpectomy scar site developing asymmetry on imaging.

\section{ABBREVIATIONS}

CC: Craniocaudal

ML: Mediolateral

MLO: Mediolateral Oblique

Post op: Post-operative

\section{KEYWORDS}

BioZorb; breast cancer recurrence; fat necrosis; foreign body reaction; mammogram; ultrasound; MRI

\section{Online access}

This publication is online available at: www.radiologycases.com/index.php/radiologycases/article/view/3911

\section{Peer discussion}

Discuss this manuscript in our protected discussion forum at: www.radiolopolis.com/forums/JRCR

\section{Interactivity}

This publication is available as an interactive article with scroll, window/level, magnify and more features.

Available online at www.RadiologyCases.com

\section{Published by EduRad}

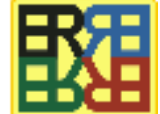

www.EduRad.org 